EPJ Web of Conferences 66, 10005 (2014)

DOI: 10.1051/epjconf/ 20146610005

(C) Owned by the authors, published by EDP Sciences, 2014

\title{
A Prototype Scintillating-Fibre Tracker for the Cosmic-ray Muon Tomography of Legacy Nuclear Waste Containers
}

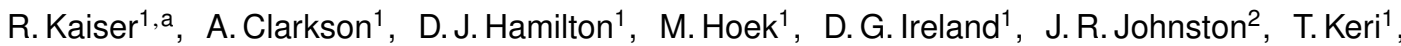 \\ S. Lumsden ${ }^{1}$, D. F. Mahon ${ }^{1}$, B. McKinnon ${ }^{1}$, M. Murray ${ }^{1}$, S. Nutbeam-Tuffs ${ }^{1}$, C. Shearer ${ }^{2}$, \\ C. Staines ${ }^{2}$, G. Yang ${ }^{1}$, and C. Zimmerman ${ }^{2}$ \\ ${ }^{1}$ Nuclear Physics Group, University of Glasgow, University Avenue, Glasgow, G12 8QQ, Scotland, UK \\ ${ }^{2}$ National Nuclear Laboratory, Central Laboratory, Sellafield, Seascale, Cumbria, CA20 1PG, England, UK
}

\begin{abstract}
Cosmic-ray muons are highly-penetrative charged particles observed at sea level with a flux of approximately $1 \mathrm{~cm}^{-2} \mathrm{~min}^{-1}$. They interact with matter primarily through Coulomb scattering which can be exploited in muon tomography to image objects within industrial nuclear waste containers. This paper presents the prototype scintillating-fibre detector developed for this application at the University of Glasgow. Experimental results taken with test objects are shown in comparison to results from GEANT4 simulations. These results verify the simulation and show discrimination between the low, medium and high- $Z$ materials imaged.
\end{abstract}

\section{Introduction}

Muons occur naturally from the decay of pions and kaons produced in cosmic ray showers in the Earth's atmosphere. Their typical momentum is in the range of several $\mathrm{GeV} \mathrm{c}^{-1}$. Cosmic muons are highly-penetrating particles which interact only weakly with matter via ionising interactions with atomic electrons and Coulomb scattering off nuclei. They are observed at sea level with a flux of approximately one muon per square centimetre per minute. These properties can be exploited in muon tomography (MT) to image the internal composition of shielded structures which would otherwise block more conventional forms of imaging radiation e.g. X-rays. Historically, radiographic techniques using cosmic-ray muons have been employed in a wide range of applications, from the first reported use in measuring the thickness of the ice burden above the Guthega-Munyang tunnel in Australia by George in the 1950s [1] and Alvarez's search for hidden chambers in the Second Pyramid of Chephren in Egypt [2] a decade later, to more recent uses in volcanology [3, 4] and nuclear threat detection for national security $[5,6]$.

Seminal work in Ref. [5] by Borozdin et al. revealed, for the first time, the potential to utilise the Coulomb scattering of muons to identify high- $Z$ materials within shielded containers. In the case of Coulomb scattering, the identification of the initial and scattered muon trajectories allows the scattering density $\lambda$ and location to be determined. Here, $\lambda$ is known to exhibit an inherent dependence on the atomic number $Z$ of the scattering material [7].

\footnotetext{
a e-mail: Ralf.Kaiser@glasgow.ac.uk
} 
At the Sellafield facility in the north-west of England, intermediate (ILW) and high-level waste products are stored in highly-engineered containment structures. In particular, there are significant quantities of ILW stainless-steel containers in storage at the Sellafield site. Muon tomography is a promising technique for characterising the content of these legacy waste containers to assist in mitigating the risk inherent with the long-term storage of materials of this nature.

\section{The Prototype Glasgow MT Detector}

For this industrial application, a prototype muon detector system has been developed and fabricated by the Nuclear Physics group at the University of Glasgow in collaboration with the UK National Nuclear Laboratory. This system consists of four scintillating-fibre tracker modules, two situated above and two below the active volume (Figure 1). Each module comprises two orthogonal planes of Saint-Gobain ${ }^{\circledR}$ BCF-10 plastic scintillating fibres with $2 \mathrm{~mm}$ pitch. One detection plane contains a single layer of 128 fibres optically-bonded onto low- $Z$, machine-grooved Rohacell ${ }^{\circledR}$ support sheets. Per detection layer, the fibres are read out by one Hamamatsu H8500 MAPMT, with a 64-pixel anode array, i.e. two fibres per pixel.
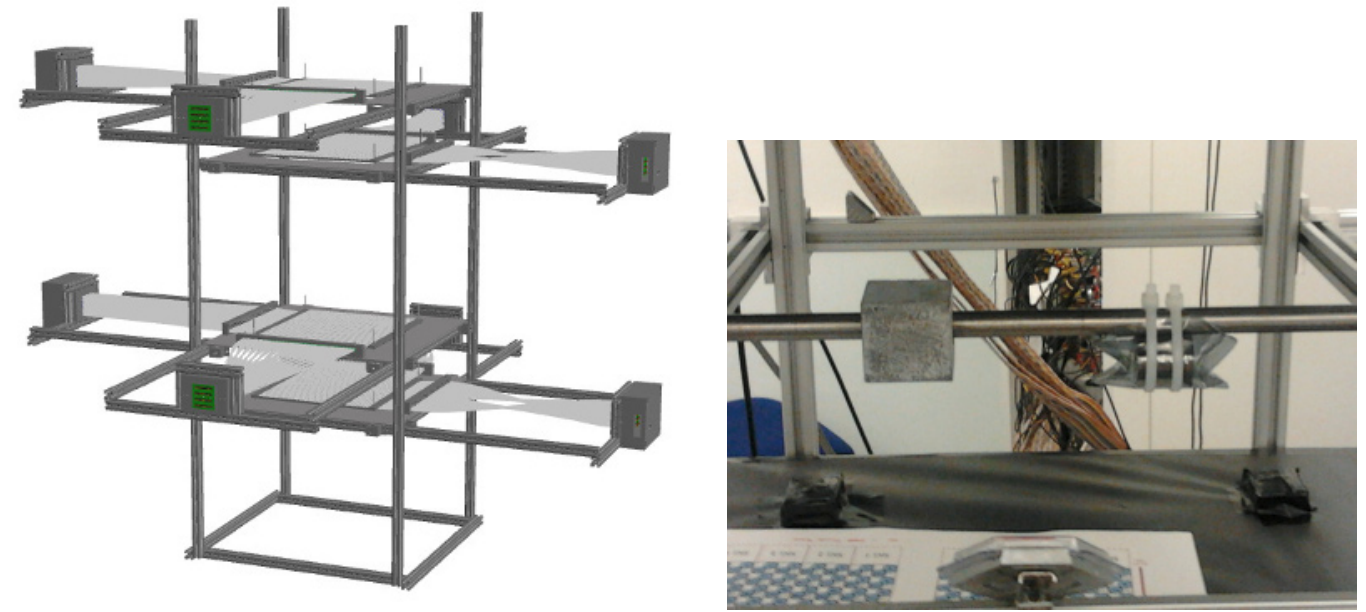

Figure 1. Left: CAD model of the prototype Glasgow MT detector. Shown are the four tracking modules consisting of two orthogonal layer of scintillating fibres each connected to a PMT. Right: Test configuration of materials placed in the central region of the assay volume comprising a $40 \mathrm{~mm}$ cube of lead (left i.e. the negative $y$-direction) surrounding a $12 \mathrm{~mm}$ diameter stainless steel cylindrical rod and a $30 \mathrm{~mm}$ long, $20 \mathrm{~mm}$ diameter cylinder of machined uranium metal (right i.e. the positive $y$-direction) suspended below.

\section{Detector Performance Studies}

Data were collected in 2012 with empty active volume for initial commissioning and alignment purposes. A multi-fold trigger on the Dynode-12 signals from the PMTs was implemented throughout this collection period to ensure that a single muon had passed through the full acceptance of the detector system. Studies revealed only minor misalignments between detector modules of less than $5 \mathrm{~mm}$ per module. Corrections to account for these were applied in the analysis software. Throughout the 
commissioning process, and subsequent data-taking period, the performance of the detector was found to be extremely stable with optimal detection efficiencies in the region of $80 \%$ for a single layer. Low multiplicities ( $<1.5$ pixel clusters on average) on each PMT were observed for candidate events. As is to be expected, minor variations in these properties were observed across the eight detection layers.

\section{Data Analysis}

An image reconstruction algorithm based on the probabilistic Maximum Likelihood Expectation Maximisation (MLEM) method introduced in Ref. [8] by Schultz et al. was further developed for this application using an accurate Geant4 [9] simulation of the prototype detector setup. For each muon, the incoming and scattered vectors are first projected to their Point of Closest Approach (PoCA). The MLEM method then calculates a normalised scattering probability in every voxel that the muon is determined to have passed through. After many muons, the most likely scattering density in each voxel is determined. Data-taking commenced in 2012 with a test configuration of objects inside the active volume as shown on the right side of Figure 1. A standalone Monte Carlo muon event generator was developed using well-defined characteristics of cosmic-ray muons including a sea-level flux of $1 \mathrm{~cm}^{-2} \mathrm{~min}^{-1}$, a mean momentum ( $\mathrm{p}_{\text {mean }}$ ) of $3.35 \mathrm{GeV} \mathrm{c}^{-1}$ with a $\mathrm{p}^{-2.7}$ slope at higher values, and a $\cos ^{2} \theta$ angular dependence. This distribution of muons was propagated through an accurate simulation, using the Geant4 framework incorporating all relevant muonic interactions with matter, of the prototype detector system and test configuration of materials shown in Figure 1.

\section{Results}

First image reconstruction results from experimental data taken using this prototype detector system are presented in Figure 2 in comparison to the corresponding simulation data for the same geometry and duration. In both the results from experimental and simulated data, sensitivity to atomic number $Z$ and discrimination between the $\lambda$ values of the stainless-steel bar, the two high- $Z$ materials, and the surrounding air is observed.
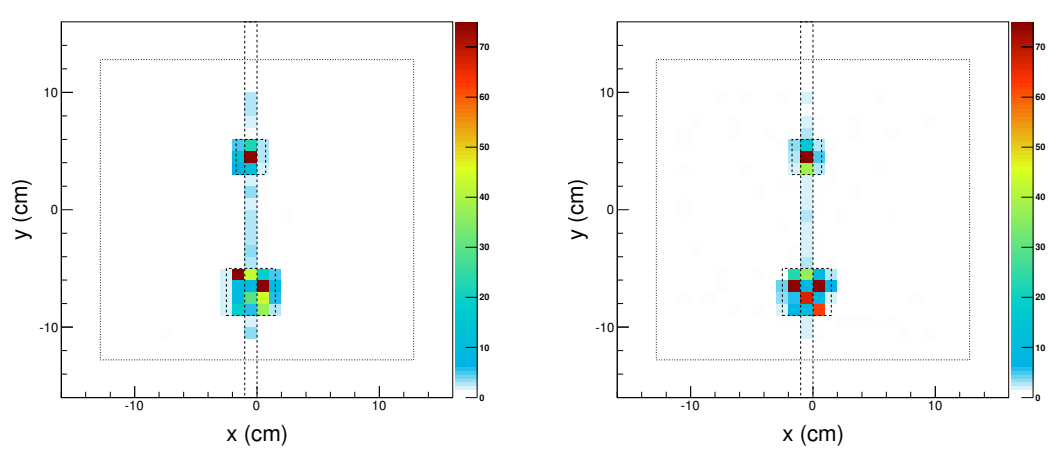

Figure 2. Comparison between images reconstructed from several weeks of exposure to cosmic-ray muons for experimental data (left) and Geant 4 simulation (right). Shown is a $1 \mathrm{~cm}$ slice in the $x y$-plane i.e. parallel to the detector modules. The dashed lines provide an estimate of the location and dimensions of the test objects in the assay volume. 
The non-uniformity of the $\lambda$ values of the reconstructed high- $Z$ objects is attributed to a combination of possible factors; a spread in $\lambda$ values for high-density materials as a result of increased Coulomb scattering (here, the PoCA input to the MLEM method reconstructs only the average scatter) and non-uniform voxel coverage of the objects (e.g. for the uranium object, the central voxel with a reconstructed $\lambda$ in excess of $70 \mathrm{mrad}^{2} \mathrm{~cm}^{-1}$ is assumed to fully occupy the uranium, whereas the surrounding voxels occupy a combination of uranium and air which acts to dilute the reconstructed $\lambda$ value).

In these tomograms, the high image-resolution of this detector system in the $x y$-plane is observed. Smearing and stretching of the image in the $z$-direction is also noted, though not presented. This is an inherent effect associated with the reconstruction of the scattering location in the principle axis of two near-parallel tracks, and is artificially exaggerated in this work by the small angular acceptance of the prototype system. Studies which address this issue are the subject of ongoing work and as such will not be described here, other than to highlight the consistency with expected results from simulation.

\section{Summary}

A prototype muon tomography system, consisting of four scintillating-fibre tracker modules, has been constructed at the University of Glasgow to assess the potential of this technology in the identification and characterisation of high- $Z$ materials stored within legacy nuclear waste containers. First results from the project, presented in this work, have confirmed the high- $Z$ material detection capabilities of this detector system which have verfied the results of promising, initial simulation studies. Discrimination between low (air), medium (stainless steel) and high- $Z$ (lead and uranium) materials was observed with several weeks of cosmic-ray muon exposure. This timescale however, is not indicative of the expected exposure time to obtain initial high $Z$ material identification.

Studies are ongoing using the small-scale prototype in Glasgow. The results of these will influence the design and construction of a large-scale detector system which will be capable of accommodating an ILW container for assay. It is foreseen that with further development, this system will be employed in the future to assay the contents of legacy nuclear waste containers at Sellafield, and in doing so, significantly impact upon future storage policy by helping to mitigate the risks inherent with the longterm storage of nuclear waste.

\section{Acknowledgements}

The author gratefully acknowledges the organising committee of the International Nuclear Physics Conference (INPC2013) for the opportunity to present this work, and Sellafield Ltd., on behalf of the UK Nuclear Decommissioning Authority, for their continued funding of this project.

\section{References}

[1] E. P. George, Commonwealth Engineer, 455 (1955)

[2] L. W. Alvarez, Science 167, 832 (1970)

[3] H. K. M. Tanaka et al., Nucl. Instrum. Meth. A555, 164 (2005)

[4] F. Fehr et al. J. Phys.: Conf. Ser. 375052019 (2012)

[5] K. Borozdin et al., Nature 422, 277 (2003)

[6] K. Gnanvo et al., IEEE Nuclear Science Symposium Conference Record, 1278 (2008)

[7] L. Schultz et al., Nucl. Instrum. Meth. A519, 687 (2004)

[8] L. Schultz et al., IEEE Transactions on Image Processing 16, 1985 (2007)

[9] S. Agostinelli et al., Nucl. Instrum. Meth. A506, 250 (2003) 